\title{
El niño cojo, pintura barroca de José de Ribera: diferente perspectiva
}

\author{
The lame boy, baroque painting by José de Ribera: a different perspective
}

Luis Gerardo Domínguez Carrillo*

Citar como: Domínguez CLG. El niño cojo, pintura barroca de José de Ribera: diferente perspectiva. Acta Med Grupo Angeles. 2021; 19 (3): 401-403. https://dx.doi.org/10.35366/101738

El barroco se enmarca en el siglo XVII, en el ámbito artístico, España vivió una de las épocas de mayor esplendor que pasaría a la historia como el Siglo de Oro. El arte proliferó gracias a la fascinación europea por la cultura española, dando importantes referentes en múltiples disciplinas como: Miguel de Cervantes, Quevedo, Góngora o Lope de Vega en la literatura, así como Diego Velázquez y José de Ribera, en pintura. Las pinturas barrocas poseen el gusto por el realismo y el naturalismo, muy alejados del canon renacentista. En dicha época, las cortes reales se rodeaban de personas con diferencias notables para aumentar su sensación de superioridad. Los autores logran proveer de humanidad a los personajes retratados sin tener en cuenta su condición. José de Ribera, apodado Lo Spagnoletto (El Españolito), fue un gran referente en este ámbito, creando obras como "El niño cojo", también llamado "El pie varo" o "El patizambo" que presenta gran atención al detalle, tratando sus rasgos como las diferencias físicas o mentales con gran dignidad. ${ }^{1}$ Una de las características del barroco es que las pinturas adquieren un tinte dinámico, rompiendo con la rigidez clásica gracias al empleo de diagonales y formas asimétricas; la variedad en cuanto a colores es otro factor determinante que, si bien en muchos casos era oscura por la influencia del tenebrismo, aporta gran dramatismo a las escenas. Durante las últimas etapas de su carrera, el artista José Ribera se vio influido por la pintura veneciana y el clasicismo, tornando su paleta más luminosa y su pintura menos dramática y más monumental. A esta etapa pertenece la obra que aquí se presenta.
En cuanto a la obra en sí, "El pie varo", también conocido como "El patizambo" o "El lisiado", es una de las pinturas más conocidas del pintor. Se anota que antes de ingresar en las colecciones del Louvre se le llamó "El enano", pues el personaje representado lo parece. Está realizada en óleo sobre tela, y fue pintada en 1642; mide $164 \mathrm{~cm}$ de alto y $92 \mathrm{~cm}$ de ancho, siendo un ejemplo del crítico realismo de la escuela española del Siglo de Oro. Se exhibe en el Museo del Louvre de París, gracias al legado del coleccionista Louis La Caze, en 1869.

El cuadro representa a un niño mendigo napolitano, de pie, frente a un cielo claro y luminoso, Ilenando el lienzo con una luz casi natural; tiene un pie deforme, varo, de manera que no puede sostenerse sobre el talón. El niño sonríe directamente al espectador, notando que le faltan algunos dientes; muestra en una mano un papel que dice en latín: DA MIHI ELIMO/SINAM PROPTER AMOREM DEI, lo que significa "Deme una limosna, por amor de Dios". Este papel era la autorización necesaria en el reino de Nápoles para ser un pordiosero; con ese mismo brazo sujeta al hombro su muleta. ${ }^{2}$

Desde el punto de vista ortopédico, se anotan las siguientes observaciones como ejercicio clínico, mencionando los puntos que a juicio del que escribe son visibles en el cuadro (Figura 1).

1. Rotación interna de ambos hombros.

2. Ambos codos en extensión (prácticamente mínimos pliegues en ropa de codo derecho (existiendo la posibilidad de no poderlos flexionar).

\footnotetext{
* Especialista en Medicina de Rehabilitación, Catedrático de la Facultad Aceptado: 27-04-2021. de Medicina de León. Universidad de Guanajuato. México.
}

Correspondencia:

Acad. Dr. Luis Gerardo Domínguez Carrillo

Correo electrónico: Igdominguez@hotmail.com

www.medigraphic.com/actamedica

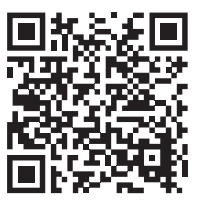


3. Posición en flexión de ambas muñecas.

4. Flexión de articulaciones metacarpo falángicas de ambas manos.

5. Flexión de interfalángicas proximales de ambas manos.

6. Ambas rodillas en extensión.

7. Hipotrofia de musculatura de pierna derecha al compararla con la contralateral que se encuentra en un plano posterior.

8. Pie derecho equino-varo aducto.

9. Caída de arco anterior de pie derecho.

10. Segundo ortejo de pie derecho en martillo, superpuesto sobre el tercer ortejo.

11. Quinto ortejo con aducción y rotación sobre su eje.

12. Descenso del arco longitudinal de pie izquierdo (pie plano).

13. Utiliza muleta con apoyo axilar, sabemos que es una muleta ya que su longitud es la misma del piso a la axila izquierda, al no tener maneral, es muy posible que el niño la manejara apoyándola en el primer espacio interóseo entre el pulgar y el índice de mano izquierda.

14. Utiliza pinza lateral del pulgar para sostener un documento.

Por lo anterior, al menos a la observación, se puede pensar como posibilidad diagnóstica en artrogriposis, y no sólo en la presencia de un pie equino-varo-aducto.

En un fragmento del artículo de Álvaro, ${ }^{3}$ intitulado "Lo neurológico y médico en la pintura barroca", se menciona:
El patizambo o El niño cojo (1642) muestra signos de espasticidad en los miembros derechos: el brazo, en extensión forzada del codo y con flexión palmar de la muñeca y dedos; la pierna, también en extensión, con apoyo solo de la punta del pie, con deformidad en varo del mismo.

Aquí habrá que anotar que esta aseveración puede prestarse a discusión, ya que ante la presencia de espasticidad, el patrón habitual es que la extremidad superior se encuentre en moderada abducción, codo en flexión, pronación de antebrazo, flexión de muñeca y pulgar atrapado por dedos en flexión, por otra parte, el patrón de espasticidad en miembro pélvico habitualmente es de aducción de cadera, rodilla en extensión y pie en equino-varo; sin embargo, como posibilidad queda en el diagnóstico diferencial.

Por otra parte, el término artrogriposis (ó $\rho$ $\theta \rho$ v 'articula-

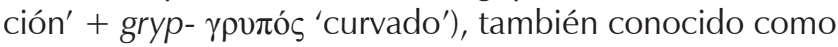
artrogriposis múltiple congénita o contracturas articulares congénitas múltiples, fue descrito por Otto como una miodistrofia en 1841; se caracteriza por contracturas articulares congénitas, no progresivas y simétricas que afectan, al menos, dos áreas diferentes del cuerpo humano. Es una enfermedad heterogénea y se han descrito cientos de padecimientos que la asocian con al menos 105 defectos genéticos. La expresión "artrogriposis" se utiliza más como un signo clínico que como un diagnóstico. ${ }^{4}$ Existen dos tipos principales de artrogriposis múltiple congénita; la más común es la amioplasia que representa $40 \%$ de los casos (tipo clásico) y ocurre en 1 de cada 10,000 nacidos vivos
Figura 1:

A) Pintura barroca de José de Ribera titulada el "Patizambo". B-D) Observaciones a nivel distal de las extremidades.
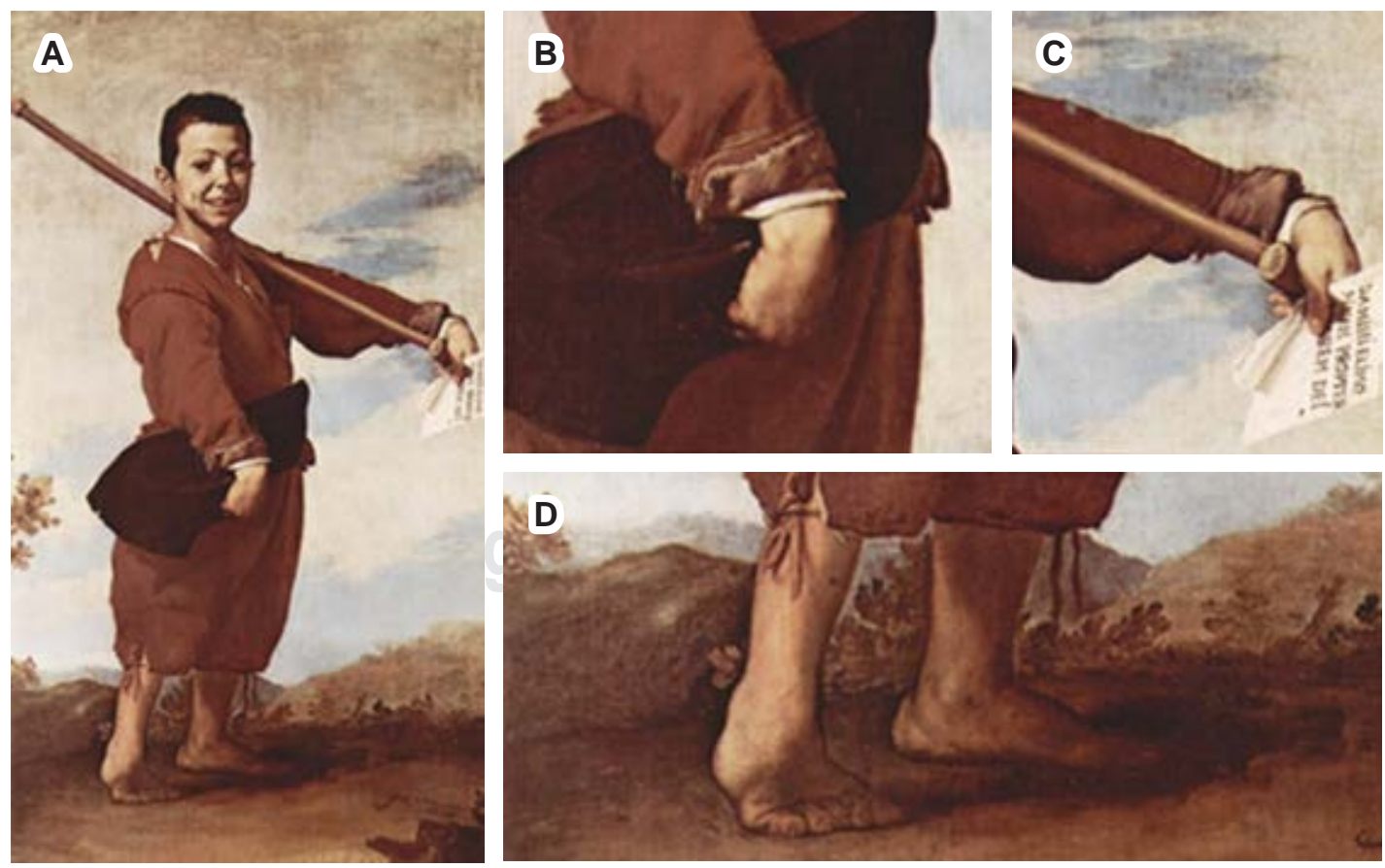
de manera esporádica. El tejido muscular es atrófico y es reemplazado por tejido graso y fibroso. Los individuos con esta forma suelen tener inteligencia normal, en estos casos las extremidades están involucradas en un patrón simétrico. La apariencia típica de la amioplasia ${ }^{5}$ se manifiesta con hombros rotados internamente y aducidos, flexión palmar y contracturas de flexión de articulaciones interfalángicas distales, luxación de cadera, contracturas de extensión de rodillas y deformidad en pie equino-varo severo. ${ }^{6}$

El segundo tipo más común es la artrogriposis distal, una alteración con herencia autosómica dominante, siendo un grupo de síndromes principalmente con contracturas articulares distales de las extremidades. Hasta la fecha se han descrito, al menos, 10 tipos diferentes de artrogriposis distal. ${ }^{7}$

Independientemente del diagnóstico, del cual como médicos sólo podemos hacer ciertas elucubraciones, al observar esta pintura, nos queda el poder admirar el arte de uno de los pintores notables del Siglo de Oro.

\section{REFERENCIAS}

1. Gardner H, Kleiner SF, Mamiya JC. Gardner's art through the ages. Belmont, CA: Thomson/Wadsworth; 2005.

2. Anguita Herrador R. El arte Barroco español. Madrid: Ediciones Encuentro; 2004.

3. Álvaro C. Lo neurológico y médico en la pintura barroca. Neurosciences and History. 2017; 5 (1): 26-37.

4. Álvarez-Quiroz P, Yokoyama-Rebollar E. Abordaje clínico y diagnóstico de la artrogriposis. Acta Pediatr Mex. 2019; 40 (1): 44-50.

5. Griffet J, Dieterich K, Bourg V, Bourgeois E. Amyoplasie et arthrogryposes distales. In: Huten D, Dohin B, Ehlinger M, Ropars M. Conférences D'enseignement 2020. Elsevier Masson; 2020. pp. 155-169. Available in: http://dx.doi.org/10.1016/b978-2-294-772504.00013-9

6. Kowalczyk B, Felus J. Arthrogryposis: an update on clinical aspects, etiology, and treatment strategies. Arch Med Sci. 2016; 12 (1): 10-24. doi: 10.5114/aoms.2016.57578.

7. Hall JG, Kimber E, Dieterich K. Classification of arthrogryposis. Am J Med Genet C Semin Med Genet. 2019; 181 (3): 300-303. doi: 10.1002/ajmg.c.31716. 